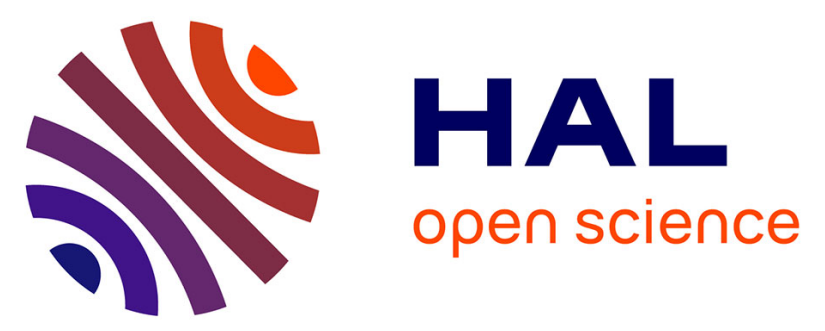

\title{
Analysis of a Model of Dormancy in Cancer as a State of Coexistence Between Tumor and Healthy Stem Cells
}

\author{
Walid Djema, Jean Clairambault, Frédéric Mazenc, Pierre Hirsch, Francois
}

Delhommeau

\section{- To cite this version:}

Walid Djema, Jean Clairambault, Frédéric Mazenc, Pierre Hirsch, Francois Delhommeau. Analysis of a Model of Dormancy in Cancer as a State of Coexistence Between Tumor and Healthy Stem Cells. ACC 2017 - American Control Conference, May 2017, Seattle, United States. pp.5135-5140, 10.23919/ACC.2017.7963751 . hal-01677927

\section{HAL Id: hal-01677927 \\ https://hal.inria.fr/hal-01677927}

Submitted on 8 Jan 2018

HAL is a multi-disciplinary open access archive for the deposit and dissemination of scientific research documents, whether they are published or not. The documents may come from teaching and research institutions in France or abroad, or from public or private research centers.
L'archive ouverte pluridisciplinaire HAL, est destinée au dépôt et à la diffusion de documents scientifiques de niveau recherche, publiés ou non, émanant des établissements d'enseignement et de recherche français ou étrangers, des laboratoires publics ou privés. 


\title{
Analysis of a Model of Dormancy in Cancer as a State of Coexistence Between Tumor and Healthy Stem Cells
}

\author{
W. Djema, \\ C. Bonnet, \\ J. Clairambault, \\ F. Mazenc, \\ P. Hirsch, \\ F. Delhommeau.
}

\begin{abstract}
We study a mathematical model of cohabitation of healthy and unhealthy stem cells, in order to address some new biological concerns. In short, we will investigate the existence and the stability properties of a positive steady state of a model that is well adapted to the study of dormancy of cancer stem cells. Mathematically, we are concerned with the analysis of a nonlinear retarded system coupled to a nonlinear differential-difference system, in the timedomain framework, via a Lyapunov-like method.
\end{abstract}

Key Words: Delay, Nonlinear, Exponential stability.

\section{INTRODUCTION}

Cells are the fundamental units that compose all living organisms [23]. A cell that engages a division process (also called cell-cycle, or proliferating phase) undergoes successive transformations until dividing into two daughter cells during the so-called mitotic M-phase (which is the last stage in the process of cell division [23]). Not all the cells are involved in proliferation, but instead, most of them are in a nondividing state, called quiescent or resting phase. Until now, the regulatory mechanism that triggers cell division and controls the committed cells, in the series of physiological actions performed during the cell-cycle, are not perfectly understood.

Stem cells (SCs) are undifferentiated cells which bring together two fascinating abilities: they constantly ensure their self-replenishment, and they are pluripotent, which means that each SC can differentiate into one among a wide range of specialized cells (see [23] for an overview on the subject, and [15] for the typical case of blood formation). Unfortunately, it happens that a pathological population of cells, that does not necessarily, initially, belong to the SCs family, acquires some self-renewing and proliferating capabilities similar to those of SCs (see [10],

\footnotetext{
Walid Djema, Catherine Bonnet and Frédéric Mazenc are with Inria and Université ParisSaclay, L2S-CentraleSupélec, Gif-sur-Yvette, France. walid.djema@inria.fr catherine.bonnet@inria.fr frederic.mazenc@l2s.centralesupelec.fr

Jean Clairambault is with Inria and Sorbonne Universités, UPMC Univ Paris 06, UMR 7598, Laboratoire Jacques-Louis Lions, Paris, France. jean.clairambault@inria.fr

Pierre Hirsch and François Delhommeau are with Groupe de Recherche Clinique sur les Myéloproliferations Aiguës et Chroniques (GRC MyPAC), Hôpital Saint-Antoine, Laboratoire d'Hématologie, Paris, France. pierre.hirsch@aphp.fr, francois.delhommeau@aphp.fr

This work is supported by ALMA3 project on the "Analysis of Acute Myeloid Leukemia », through DIGITEO, Paris-Saclay.
}

[27]). These stem-like cells are very often out of control and they can initiate (or regenerate) cancers, hence their designation as cancer stem cells (CSCs). Very often, CSCs are characterized by some unhealthy behaviors such as an excessive proliferation and an abnormal loss of differentiation faculties (this is observed, for instance, in acute myeloid leukemia). Curiously, however, in some rare cases CSCs do not overproliferate (i.e. cancer cells exist but without causing an effective disease). Such an unexpected behavior is referred to as «tumor dormancy». Strong evidence that supports the existence of tumor dormancy was established many years ago, when some microscopic tumors were discovered during autopsy examinations (see [25], [17], and the references therein). Even during their dormant state, CSCs are distinguishable through specific markers on their surfaces (see [10] and the references therein). The most likely explanations -retained for instance in [17]- of the fact that a cell which expresses cancer markers can exist in a dormancy state are: i) blood and nutrient supply issues that prevent tumor growth (or at least delay its clinical manifestation [24]), and, ii) vigilance of immune system which, in some rare cases, suffices to stop tumor development [31] (see the references in [24], [31], [17]).

Apart from the interpretation of tumor dormancy as an observed natural human phenomenon (i.e. when CSCs are maintained in a dormant state through the own body capabilities), an interest is arising from a therapeutic standpoint on how can we bring CSCs from a state of overproliferation into a dormant one? Indeed, since current treatments of cancer consist in delivering drugs at their maximum doses (i.e. tolerable quantities, generally defined according to their side-effects and toxicity thresholds) in order to totally eradicate declared tumors; and on the other hand, knowing that non-fully eradicated tumors will grow again, even more aggressively than the initial ones [10], it appears that the option of maintaining the tumor in dormancy is more promising than trying to eradicate it [16]. At this juncture, the development of a strong mathematical modeling framework appears as a fundamental tool in order to apprehend tumor dormancy as a biological mechanism, with the ultimate goal of application in cancer therapy.

The mathematical model of interest in this paper is a nonlinear time-delay system describing the behavior of healthy SCs, coupled to a nonlinear differential-difference 
system describing CSCs. From a theoretical standpoint, we know that no systematic method apply to analyze the class of nonlinear time-delay systems that we are interested in. In particular, using Lyapunov techniques, the search of a suitable Lyapunov functional may sometimes become a headache. The difficulty is even increased when the solutions are not uniformly continuous, since invariance principles or Barbalat's lemma cannot be employed to prove asymptotic stability. In the light of the above considerations, our contribution lies with the Lyapunov-based approach that we propose in order to address some biological concerns on CSCs dormancy.

\section{A MATHEMATICAL MODEL OF CSCS INVOLVING COEXISTENCE WITH HEALTHY SCS}

Based on numerous studies on the modeling and the analysis of blood cell dynamics ${ }^{1}$ (including notably the works of Mackey et al. [29], [21], [12], and, Adimy et al. [1], [2], and also the works of Marciniak-Czochra et al. [22], Özbay et al. [26], Avila et al. [4], Fridman et al. [14], Djema et al. [6], among others), we intend to make a more general study that may cover other types of SCs and CSCs in liquid or solid tumors. For that, we revisit the model proposed in [7], which describes the coexistence of healthy and mutated hematopoietic stem cells, and is in fact valid for a wide panel of stem cells. Thus, while retaining the original version of this system, we slightly modify it in order to allow the existence of tumor dormancy, that may occur for many types of CSCs (e.g. breast cancer). More precisely, we focus on the model illustrated in Figure 1, where healthy SCs (on the left) are coupled to CSCs (on the right). Here CSCs are characterized by an overproliferation ability represented by the parameter $\tilde{K}$, as introduced recently in [1], and previously envisaged in a different configuration in [4]. The initial model proposed in [7] (as well as the one in [4]) cannot be directly used to model dormancy. To overcome this issue we slightly modify the manner of coupling healthy and mutated cells.

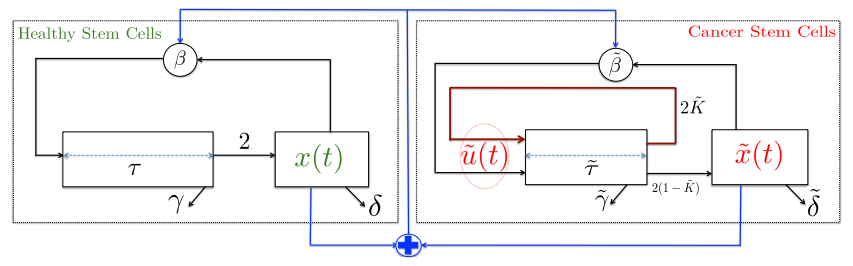

Fig. 1. Schematic representation of coupled model of healthy SCs (on the left) and CSCs (unhealthy part on the right).

Now, we present very briefly the coupled model of healthy SCs and CSCs. The interested reader is invited to refer to [1] and [7] for more details. We consider that SCs are in a resting phase (non-dividing state) or in a proliferating phase (dividing state) as many other works

\footnotetext{
${ }^{1}$ Hematopoietic stem cells, which are at the root of hematopoiesis, are certainly among the most well-studied types of stem cells, in view of their great importance in the human body.
}

(for instance [21], [29] and [2]). The total density of resting healthy SCs at time $t \geq 0$ is denoted by $x(t)$, while the total density of resting CSCs at time $t \geq 0$ is denoted by $\tilde{x}(t)$. Since CSCs proliferate more than healthy SCs, we consider that a rate $\tilde{K} \in(0,1)$ of daughter CSCs will return directly to the proliferating stage (i.e. we assume that a sub-population of cells is permanently active in the unhealthy proliferating phase [1]). This behavior is not noticed for healthy SCs (Figure 1) where daughter cells leave the proliferating compartment to join the resting one, where they can stay until their death, can differentiate or may start a new proliferating cycle. In the unhealthy compartment, $\tilde{u}(t)$ represents the density of new proliferating cells at time $t \geq 0$ (i.e. the density of new proliferating cells, which start their division cycle at time $t \geq 0$ ). Moreover, we denote by $\delta$ (resp. $\tilde{\delta})$ the rate of resting cells which is lost either by differentiation or natural cell death for healthy stem cells (resp. CSCs). A resting cell may start a cell division cycle by entering to the proliferating phase, during which each proliferating healthy SC (resp. CSC) may die by apoptosis rate $\gamma$ (resp. $\tilde{\gamma}$ ), or complete its mitosis and become two daughter cells at the end of the proliferating phase. We denote $\tau$ (resp. $\tilde{\tau}$ ) the average time taken by a healthy cell (resp. unhealthy) to complete its mitosis in the healthy (resp. unhealthy) proliferating compartment. For healthy and unhealthy cells, we assume that the passing from the resting state to the proliferating one is controlled by a reintroduction function (see [21], [29]). We consider that $\beta$ (resp. $\tilde{\beta}$ ) is the reintroduction function from the healthy (resp. unhealthy) resting phase to the healthy (resp. unhealthy) proliferating phase. Moreover, $\beta$ and $\tilde{\beta}$ are assumed to depend on both the total density of resting healthy cells $x$ and the total density of unhealthy resting cells $\tilde{x}$. More precisely, we assume that $\tilde{\beta}$ and $\beta$ are nonlinear, continuous, and decreasing functions, and that, $\lim _{m \rightarrow \infty} \tilde{\beta}(m)=\lim _{m \rightarrow \infty} \beta(m)=0$. As in $[21],[29],[2]$, and [26] we consider the typical forms:

$$
\tilde{\beta}(m)=\frac{\tilde{\beta}(0)}{1+\tilde{b} m^{\tilde{n}}}, \quad \beta(m)=\frac{\beta(0)}{1+b m^{n}}
$$

where $\tilde{b}, b, \tilde{\beta}(0)$ and $\beta(0)$ are strictly positive real numbers and $\tilde{n} \geq 2$ and $n \geq 2$. The model illustrated in Figure 1 was described in [7] by an age-structured model (see for instance [19], Chapter 5). It is a McKendrick model ([20], [12]) composed of coupled age-structured partial differential equations, with their corresponding boundary and initial conditions, that we reduced using the method of characteristics to the following delay differential-difference system [7]:

$$
\left\{\begin{aligned}
\dot{\tilde{x}}(t)= & -[\tilde{\delta}+\tilde{\beta}(x(t)+\tilde{\alpha} \tilde{x}(t))] \tilde{x}(t) \\
& +2(1-\tilde{K}) e^{-\tilde{\gamma} \tilde{\tau}} \tilde{u}(t-\tilde{\tau}) \\
\tilde{u}(t)= & \tilde{\beta}(x(t)+\tilde{\alpha} \tilde{x}(t)) \tilde{x}(t)+2 \tilde{K} e^{-\tilde{\gamma} \tilde{\tau}} \tilde{u}(t-\tilde{\tau}), \\
\dot{x}(t)= & -[\delta+\beta(\alpha x(t)+\tilde{x}(t))] x(t) \\
& +2 e^{-\gamma \tau} \beta(\alpha x(t-\tau)+\tilde{x}(t-\tau)) x(t-\tau) .
\end{aligned}\right.
$$


In fact, in [7] it was considered that $(\alpha, \tilde{\alpha})=(1,1)$. This is the simplest way of coupling healthy and unhealthy cells, in which a homogeneous sensitivity (see [8]) of the functions $\beta$ and $\tilde{\beta}$ with respect to the total density of healthy and unhealthy cells is assumed. In this paper, we study a slightly more general framework by considering the case in which $(\alpha, \tilde{\alpha})=(1, \tilde{\alpha})$, where $\tilde{\alpha}>0$ is a free parameter. We can prove that a unique piecewise continuous solution $(\tilde{x}(t), \tilde{u}(t), x(t))$ exists for all $t \geq 0$, when system (2) is associated with appropriate initial conditions $\left(\varphi_{\tilde{x}}, \varphi_{\tilde{u}}, \varphi_{x}\right)$ such that $\varphi_{\tilde{x}} \in \mathcal{C}([-\tau, 0], \mathbb{R})$, $\varphi_{x} \in \mathcal{C}([-\tau, 0], \mathbb{R})$ and $\varphi_{\tilde{u}} \in \mathcal{C}([-\tilde{\tau}, 0], \mathbb{R})$. In addition, we can show that system (2) is positive since $\tilde{K} \in$ $(0,1)$. Throughout this work only positive solutions of (2) are considered. Next, we notice that $(0,0,0)$ is an equilibrium point of the studied model. Biologically, it means the extinction of all the healthy and unhealthy blood cells, which is not the best expected scenario. On the other hand, from a therapeutic standpoint, the common approach is to eradicate unhealthy cells while ensuring that healthy cells survive. That is exactly what we did in [7], where we analyzed a steady state in the form $\left(0,0, x_{e}>0\right)$. The present paper will be devoted to the scenario where healthy and unhealthy cells survive and coexist in a prolonged dormancy state.

\section{MAIN RESUlts}

\section{A. Notable features of the studied model}

Escape from dormancy in diseased cells: One of the main concerns in the theoretical analysis of dormancy is to explain how escape from tumor dormancy can emerge [17]. It was also the motivation to introduce the model in [1], where the authors were looking for unbounded solutions to explain unlimited proliferation of CSCs. Based on their results, we emphasize the fact that unbounded solutions in CSCs compartment may occur. Obviously, from the second equation in (2), we observe that when $2 \tilde{K} e^{-\tilde{\gamma} \tilde{\tau}}>0$, we obtain, $\lim _{t \rightarrow+\infty} \tilde{u}(t)=+\infty$. A direct consequence from the the first equation in (2) is that $\lim _{t \rightarrow+\infty} \tilde{x}(t)=+\infty$ (it can be proved by contradiction, as previously done in [1]). This situation reflects the escape from dormancy of tumor cells (solid tumors), or the invasion of the bone marrow by blasts (as an example of liquid tumors).

Existence of a unique dormancy steady state $\mathfrak{D}$ Now, we go forward in the analysis by first discussing the conditions that make possible the existence of dormancy. Let $\mathfrak{D}=\left(\tilde{x}_{e}, \tilde{u}_{e}, x_{e}\right)$ be a steady state of $(2)$, then

$$
\left\{\begin{array}{l}
{\left[\tilde{\delta}+\tilde{\beta}\left(x_{e}+\tilde{\alpha} \tilde{x}_{e}\right)\right] \tilde{x}_{e}=2(1-\tilde{K}) e^{-\tilde{\gamma} \tilde{\tau}} \tilde{u}_{e}} \\
\tilde{\beta}\left(x_{e}+\tilde{\alpha} \tilde{x}_{e}\right) \tilde{x}_{e}=\left(1-2 \tilde{K} e^{-\tilde{\gamma} \tilde{\tau}}\right) \tilde{u}_{e} \\
{\left[\delta-\left(2 e^{-\gamma \tau}-1\right) \beta\left(x_{e}+\tilde{x}_{e}\right)\right] x_{e}=0}
\end{array}\right.
$$

since $(\alpha, \tilde{\alpha})=(1, \tilde{\alpha})$. We want to determine necessary and sufficient conditions for the existence of $\mathfrak{D}$ where $x_{e}>0, \tilde{x}_{e}>0$ and $\tilde{u}_{e}>0$. First, since $\beta$ is continuous and decreasing from $\beta(0)$ to zero, we deduce from the third equation in (3) that

$$
\delta<\left[2 e^{-\gamma \tau}-1\right] \beta(0),
$$

is a necessary condition for the existence of $x_{e}$ and $\tilde{x}_{e}$ such that $x_{e}+\tilde{x}_{e}>0$, and therefore it is a necessary condition for the existence of $\mathfrak{D}$. Next, from the second equation in (3), we have $\tilde{u}_{e}=\frac{\tilde{\beta}\left(x_{e}+\tilde{\alpha} \tilde{x}_{e}\right) \tilde{x}_{e}}{1-2 \tilde{K} e^{-\tilde{\gamma} \tilde{\tau}}}$. By substituting $\tilde{u}_{e}$ in the first equation of (3), we get

$$
\left[\tilde{\delta}-\frac{2 e^{-\tilde{\gamma} \tilde{\tau}}-1}{1-2 \tilde{K} e^{-\tilde{\gamma} \tilde{\tau}}} \tilde{\beta}\left(x_{e}+\tilde{\alpha} \tilde{x}_{e}\right)\right] \tilde{x}_{e}=0
$$

The fact that $\tilde{\beta}$ is continuous and decreasing implies that

$$
\tilde{\delta}<\left[\frac{2 e^{-\tilde{\gamma} \tilde{\tau}}-1}{1-2 \tilde{K} e^{-\tilde{\gamma} \tilde{\tau}}}\right] \tilde{\beta}(0),
$$

is a necessary condition for the existence of $x_{e}$ and $\tilde{x}_{e}$ such that $x_{e}+\tilde{\alpha} \tilde{x}_{e}>0$. Therefore it is also a necessary condition for the existence of $\mathfrak{D}$.

Obviously, (4), (6) and $\tilde{u}_{e}=\frac{\tilde{\beta}\left(x_{e}+\tilde{\alpha} \tilde{x}_{e}\right) \tilde{x}_{e}}{1-2 \tilde{K} e^{-\tilde{\gamma} \tilde{\tau}}}$ imply that $1<2 e^{-\gamma \tau}$, and $2 \tilde{K} e^{-\tilde{\gamma} \tilde{\tau}}<1<2 e^{-\tilde{\gamma} \tilde{\tau}}$.

Now, let us focus on the case where $x_{e}>0$ and $\tilde{x}_{e}>0$. If the conditions (4) and (6) are satisfied, then necessarily

$$
\left\{\begin{array}{l}
x_{e}+\tilde{\alpha} \tilde{x}_{e}=\tilde{\beta}^{-1}\left(\tilde{\delta} \frac{1-2 \tilde{K} e^{-\tilde{\gamma} \tilde{\tau}}}{2 e^{-\tilde{\gamma} \tilde{\tau}}-1}\right) \\
x_{e}+\tilde{x}_{e}=\beta^{-1}\left(\frac{\delta}{2 e^{-\gamma \tau}-1}\right) .
\end{array}\right.
$$

We deduce that

$$
\left\{\begin{array}{l}
x_{e}=\frac{1}{\tilde{\alpha}-1}\left[\tilde{\alpha} \beta^{-1}(\mu)-\tilde{\beta}^{-1}(\tilde{\mu})\right], \\
\tilde{x}_{e}=\frac{1}{\tilde{\alpha}-1}\left[\tilde{\beta}^{-1}(\tilde{\mu})-\beta^{-1}(\mu)\right] \\
\tilde{u}_{e}=\frac{\tilde{\delta}}{2 e^{-\tilde{\gamma} \tilde{\gamma}}-1} \tilde{x}_{e}
\end{array}\right.
$$

where, $\mu=\frac{\delta}{2 e^{-\gamma \tau}-1}$, and, $\tilde{\mu}=\frac{\tilde{\delta}\left(1-2 \tilde{K} e^{-\tilde{\gamma} \tilde{\tau}}\right)}{2 e^{-\tilde{\gamma} \tilde{\tau}}-1}$.

The case $\tilde{\alpha}=1$ : In this situation, (7) gives

$$
\left\{\begin{array}{l}
x_{e}+\tilde{x}_{e}=\tilde{\beta}^{-1}(\tilde{\mu})=\beta^{-1}(\mu), \\
\tilde{u}_{e}=\frac{\tilde{\delta}}{2 e^{-\tilde{\gamma} \tilde{\tau}}-1} \tilde{x}_{e} .
\end{array}\right.
$$

This case corresponds to a continuum equilibrium points that we want to avoid in this work.

The case $\tilde{\alpha}>1$ or $\tilde{\alpha}<1$ : From (8), we deduce that a necessary condition for the existence of $\mathfrak{D}$ when $\tilde{\alpha}<1$ is given by: $\tilde{\alpha} \beta^{-1}(\mu)<\tilde{\beta}^{-1}(\tilde{\mu})<\beta^{-1}(\mu)$. Similarly, a necessary condition for the existence of $\mathfrak{D}$ when $\tilde{\alpha}>1$ is $\beta^{-1}(\mu)<\tilde{\beta}^{-1}(\tilde{\mu})<\tilde{\alpha} \beta^{-1}(\mu)$.

We summarize the above discussion as follows:

Proposition 1: If the conditions

$$
\mu<\beta(0), \tilde{\mu}<\tilde{\beta}(0), 2 \tilde{K} e^{-\tilde{\gamma} \tilde{\tau}}<1<2 e^{-\tilde{\gamma} \tilde{\tau}},
$$

and,

$$
\left\{\begin{array}{l}
\tilde{\alpha} \beta^{-1}(\mu)<\tilde{\beta}^{-1}(\tilde{\mu})<\beta^{-1}(\mu), \text { for } \tilde{\alpha} \in(0,1), \\
\beta^{-1}(\mu)<\tilde{\beta}^{-1}(\tilde{\mu})<\tilde{\alpha} \beta^{-1}(\mu), \text { for } \tilde{\alpha}>1
\end{array}\right.
$$

where $\mu$ and $\tilde{\mu}$ are defined in (9), are satisfied, then a unique strictly positive steady state $\mathfrak{D}=\left(\tilde{x}_{e}, \tilde{u}_{e}, x_{e}\right)$, 
exists and is given by (8).

Remark 1: i) Uniqueness in Proposition 1 means that a unique isolated strictly positive equilibrium point $\mathfrak{D}$ exists, but it is not the unique equilibrium point of the system (2), since the origin and the points $\left(0,0, x_{e}\right)$, $\left(\tilde{x}_{e}, \tilde{u}_{e}, 0\right)$ are also steady states of system (2).

ii) The third condition in (11) expresses a relationship between the fast-self renewing ability $\tilde{K}$, the apoptosis rate of malignant cells $\tilde{\gamma}$ and their cell-cycle duration $\tilde{\tau}$. We notice that even if $\tilde{K}$ is relatively important, and knowing that it is not easy to act on $\tilde{K}$ by drugs infusion, it is still possible to guarantee the existence of dormancy by increasing $\tilde{\tau} \tilde{\gamma}$. However, the increase must be moderate to not exceed the upper-bound $\tilde{\gamma} \tilde{\tau}<\ln (2)$.

\section{B. Stability analysis of the dormancy steady state}

1) A new representation of the system: We want to investigate the stability properties of $\mathfrak{D}$ when it exists. Thus, we assume that the conditions given in Proposition 1 are satisfied and we perform the classical changes of coordinates $\tilde{X}=\tilde{x}-\tilde{x}_{e}, \tilde{U}=\tilde{u}-\tilde{u}_{e}$, and $X=x-x_{e}$. Then, it follows from (2) that

$$
\left\{\begin{aligned}
& \dot{\tilde{X}}(t)=-\left[\tilde{\delta}+\tilde{\beta}\left(X(t)+\tilde{\alpha} \tilde{X}(t)+x_{e}+\tilde{\alpha} \tilde{x}_{e}\right)\right]\left(\tilde{X}(t)+\tilde{x}_{e}\right) \\
&+2(1-\tilde{K}) e^{-\tilde{\gamma} \tilde{\tau}}\left(\tilde{U}(t-\tilde{\tau})+\tilde{u}_{e}\right) \\
& \tilde{u}(t)=\tilde{\beta}\left(X(t)+\tilde{\alpha} \tilde{X}(t)+x_{e}+\tilde{\alpha} \tilde{x}_{e}\right)\left(\tilde{X}(t)+\tilde{x}_{e}\right) \\
&+2 \tilde{K} e^{-\tilde{\gamma} \tilde{\tau}}\left(\tilde{U}(t-\tilde{\tau})+\tilde{u}_{e}\right) \\
& \dot{X}(t)=-[\left.\delta+\beta\left(X(t)+\tilde{X}(t)+x_{e}+\tilde{x}_{e}\right)\right]\left(X(t)+x_{e}\right) \\
&+ 2 e^{-\gamma \tau} \beta\left(X(t-\tau)+\tilde{X}(t-\tau)+x_{e}+\tilde{x}_{e}\right) \\
& \times\left(X(t-\tau)+x_{e}\right)
\end{aligned}\right.
$$

To ease the analysis of the above system, we rewrite it in a more convenient form. Observe that for all $\mathfrak{z}>-\mathfrak{e}$, $\mathfrak{e}>0$, where, $\mathfrak{z}=X+\tilde{X}$ and $\mathfrak{e}=x_{e}+\tilde{x}_{e}$, we have (with an abuse of notation),

$$
\beta(\mathfrak{z}+\mathfrak{e})=\beta(\mathfrak{e})+\theta \mathfrak{z}+R(\mathfrak{z}),
$$

where $\beta$ is the function defined in (1), and $\theta=\beta^{\prime}(\mathfrak{e})$, $R(\mathfrak{z})=\int_{\mathfrak{e}}^{\mathfrak{e}+\mathfrak{z}}(\mathfrak{z}+\mathfrak{e}-\ell) \beta^{(2)}(\ell) d \ell$.

Next, for all $\tilde{\mathfrak{z}}>-\tilde{\mathfrak{e}}, \tilde{\mathfrak{e}}>0$, where, $\tilde{\mathfrak{z}}=X+\tilde{\alpha} \tilde{X}$, and, $\tilde{\mathfrak{e}}=x_{e}+\tilde{\alpha} \tilde{x}_{e}$, we get similarly to (13),

$$
\tilde{\beta}(\tilde{\mathfrak{z}}+\tilde{\mathfrak{e}})=\tilde{\beta}(\tilde{\mathfrak{e}})+\tilde{\theta} \tilde{\mathfrak{z}}+\tilde{R}(\tilde{\mathfrak{z}}),
$$

where, $\tilde{\theta}=\tilde{\beta}^{\prime}(\tilde{\mathfrak{e}})$, and $\tilde{R}(\tilde{\mathfrak{z}})=\int_{\tilde{\mathfrak{e}}}^{\tilde{\mathfrak{e}}+\tilde{\mathfrak{z}}}(\tilde{\mathfrak{z}}+\tilde{\mathfrak{e}}-\ell) \tilde{\beta}^{(2)}(\ell) d \ell$. Therefore, using (13)-(14), and by simplifying some terms using (3), we get

$$
\left\{\begin{aligned}
\dot{\tilde{X}}(t)= & -\mathfrak{a}_{1} \tilde{X}(t)-\mathfrak{a}_{2} X(t)+\mathfrak{a}_{3} \tilde{U}(t-\tilde{\tau}) \\
& +F(X(t), \tilde{X}(t)) \\
\tilde{U}(t)= & \mathfrak{a}_{4} \tilde{X}(t)+\mathfrak{a}_{2} X(t)+\mathfrak{a}_{5} \tilde{U}(t-\tilde{\tau}), \\
& -F(X(t), \tilde{X}(t)) \\
\dot{X}(t)= & -\mathfrak{a}_{6} X(t)-\mathfrak{a}_{7} \tilde{X}(t)+\mathfrak{a}_{8} X(t-\tau) \\
& +\mathfrak{a}_{9} \tilde{X}(t-\tau)+G\left(X_{t}, \tilde{X}_{t}\right),
\end{aligned}\right.
$$

where

$$
\begin{aligned}
F(X(t), \tilde{X}(t)) & \left.=-\tilde{\theta}\left[\tilde{\alpha} \tilde{X}^{2}(t)\right)+X(t) \tilde{X}(t)\right] \\
& -\tilde{R}(X(t)+\tilde{\alpha} \tilde{X}(t))\left(\tilde{X}(t)+\tilde{x}_{e}\right),
\end{aligned}
$$

and,

$$
\begin{aligned}
& G\left(X_{t}, \tilde{X}_{t}\right)=-\theta\left[X^{2}(t)+X(t) \tilde{X}(t)\right] \\
& -R(X(t)+\tilde{X}(t))\left(X(t)+x_{e}\right) \\
& +2 e^{-\gamma \tau} \theta\left[X^{2}(t-\tau)+X(t-\tau) \tilde{X}(t-\tau)\right] \\
& +2 e^{-\gamma \tau} R(X(t-\tau)+\tilde{X}(t-\tau)) \\
& \quad \times\left(X(t-\tau)+x_{e}\right),
\end{aligned}
$$

and where the constants parameters are given by

$$
\left\{\begin{array}{l}
\mathfrak{a}_{1}=\tilde{\delta}+\tilde{\beta}\left(x_{e}+\tilde{\alpha} \tilde{x}_{e}\right)+\tilde{\alpha} \tilde{\theta} \tilde{x}_{e}, \quad \mathfrak{a}_{2}=\tilde{\theta} \tilde{x}_{e} \\
\mathfrak{a}_{3}=2(1-\tilde{K}) e^{-\tilde{\gamma} \tilde{\tau}}, \mathfrak{a}_{4}=\tilde{\beta}\left(x_{e}+\tilde{\alpha} \tilde{x}_{e}\right)+\tilde{\alpha} \tilde{\theta} \tilde{x}_{e} \\
\mathfrak{a}_{5}=2 \tilde{K} e^{-\tilde{\gamma} \tilde{\tau}}, \quad \mathfrak{a}_{6}=\delta+\beta\left(x_{e}+\tilde{x}_{e}\right)+\theta x_{e} \\
\mathfrak{a}_{7}=\theta x_{e}, \quad \mathfrak{a}_{8}=2 e^{-\gamma \tau}\left[\beta\left(x_{e}+\tilde{x}_{e}\right)+\theta x_{e}\right] \\
\mathfrak{a}_{9}=2 e^{-\gamma \tau} \theta x_{e}
\end{array}\right.
$$

We notice that if the trajectories of (15) converge exponentially to the origin, then the positive trajectories of the system (2) converge exponentially to $\mathfrak{D}$. Now, we state the following assertions which give sector conditions on the nonlinear terms $R, F$ and $G$ (the proof is omitted here). First, we can prove that there exist strictly positive constants $\mathfrak{s}, \tilde{\mathfrak{s}}, \mathfrak{m}$ and $\tilde{\mathfrak{m}}$ such that

$$
\begin{aligned}
&|R(\mathfrak{z})| \leq \mathfrak{s}|\mathfrak{z}|, \text { and }|\tilde{R}(\tilde{\mathfrak{z}})| \leq \tilde{\mathfrak{s}}|\tilde{\mathfrak{z}}|, \\
&|R(\mathfrak{z})| \leq \mathfrak{m} \mathfrak{z}^{2}, \quad \text { and }|\tilde{R}(\tilde{\mathfrak{z}})| \leq \tilde{\mathfrak{m}} \tilde{\mathfrak{z}}^{2},
\end{aligned}
$$

for all $\mathfrak{z}>-\mathfrak{e}(\mathfrak{z}$ and $\mathfrak{e}$ are defined before (13)), and for all $\tilde{\mathfrak{z}}>\tilde{\mathfrak{e}}(\tilde{\mathfrak{z}}$ and $\tilde{\mathfrak{e}}$ are defined before (14)). Moreover, using (19) and (20), we can determine strictly positive constants $\mathfrak{c}_{i}, i=\{1, \ldots, 6\}$, such that the following quadratic upper bounds hold true:

$$
\begin{gathered}
|F(X, \tilde{X})| \leq \mathfrak{c}_{1} Q(X)+\mathfrak{c}_{2} Q(\tilde{X}), \\
\left|G\left(X_{t}, \tilde{X}_{t}\right)\right| \leq \mathfrak{c}_{3} Q(X(t))+\mathfrak{c}_{4} Q(\tilde{X}(t)) \\
+\mathfrak{c}_{5} Q(X(t-\tau))+\mathfrak{c}_{6} Q(\tilde{X}(t-\tau)) .
\end{gathered}
$$

Remark 2: Comparing the present study with [7] one notices that [7] was devoted to the study of a simpler model than the system (15) analyzed here. Indeed, the model in [7] can be obtained by putting $\tilde{\alpha}=1$ and by eliminating all the terms where $\tilde{x}_{e}$ is present in equations (15), (18), (16) and (17).

2) Obtaining Decay Conditions: We want to investigate the stability properties of the coupled system using its representation as (15), (18), (16), and (17). Due to the coordinates shifting, the trajectories of system (15) are not positive. Therefore, we introduce the following functional:

$$
\begin{aligned}
V\left(X_{t}, \tilde{X}_{t}, \tilde{U}_{t}\right)= & Q(X(t))+\lambda_{1} Q(\tilde{X}(t))+\lambda_{2} \mathcal{S}\left(X_{t}\right) \\
& +\lambda_{3} \mathcal{S}\left(\tilde{X}_{t}\right)+\lambda_{4} \mathcal{Y}\left(\tilde{U}_{t}\right)
\end{aligned}
$$


where, $Q(\ell)=\frac{\ell^{2}}{2}, \mathcal{Y}\left(\tilde{U}_{t}\right)=\int_{t-\tilde{\tau}}^{t} e^{\rho_{1}(\ell-t)} Q(\tilde{U}(\ell)) d \ell$, $\mathcal{S}\left(\tilde{X}_{t}\right)=\int_{t-\tau}^{t} e^{\rho_{2}(\ell-t)} Q(\tilde{X}(\ell)) d \ell$, and where $\lambda_{1}, \lambda_{2}, \lambda_{3}$ and $\lambda_{4}, \rho_{1}$, and $\rho_{2}$ are positive constants to be selected. Through lengthy calculations, we can prove that the time-derivative of the functional $V$ along the trajectories of (15) is given, for almost all $t \geq 0$, by:

$$
\begin{aligned}
\dot{V}(t) & =-\left[2 \lambda_{1} \mathfrak{a}_{1}-\lambda_{3}-\lambda_{4} \mathfrak{a}_{4}^{2}\right] Q(\tilde{X}(t))-\rho_{2} \lambda_{3} \mathcal{S}\left(\tilde{X}_{t}\right) \\
& -\left[2 \mathfrak{a}_{6}-\lambda_{2}-\lambda_{4} \mathfrak{a}_{2}^{2}\right] Q(X(t))-\rho_{2} \lambda_{2} \mathcal{S}\left(X_{t}\right) \\
& -\lambda_{4}\left[e^{-\rho_{1} \tilde{\tau}}-\mathfrak{a}_{5}^{2}\right] Q(\tilde{U}(t-\tilde{\tau}))-\rho_{1} \lambda_{4} \mathcal{Y}\left(\tilde{U}_{t}\right) \\
& -\lambda_{2} e^{-\rho_{2} \tau} Q(X(t-\tau))-\lambda_{3} e^{-\rho_{2} \tau} Q(\tilde{X}(t-\tau)) \\
& -\left[\mathfrak{a}_{2} \lambda_{1}+\mathfrak{a}_{7}-\lambda_{4} \mathfrak{a}_{2} \mathfrak{a}_{4}\right] X(t) \tilde{X}(t)+\mathfrak{a}_{8} X(t) X(t-\tau) \\
& +\mathfrak{a}_{9} X(t) \tilde{X}(t-\tau)+\left[\mathfrak{a}_{3} \lambda_{1}+\mathfrak{a}_{4} \mathfrak{a}_{5} \lambda_{4}\right] \tilde{X}(t) \tilde{U}(t-\tilde{\tau}) \\
& +\mathfrak{a}_{2} \mathfrak{a}_{5} \lambda_{4} X(t) \tilde{U}(t-\tilde{\tau})+\tilde{X}(t) F(X(t), \tilde{X}(t)) \\
& +X(t) G\left(X_{t}, \tilde{X}_{t}\right)-\mathfrak{a}_{5} \lambda_{4} F(X(t), \tilde{X}(t)) \tilde{U}(t-\tilde{\tau}) \\
& -\lambda_{4} F(X(t), \tilde{X}(t))\left[\mathfrak{a}_{4} \tilde{X}(t)+\mathfrak{a}_{2} X(t)\right] \\
& +\lambda_{4} Q(F(\tilde{X}(t), X(t))) .
\end{aligned}
$$

Recall that for any constants $\nu_{1}>0, \nu_{2}>0, \nu_{3}>0$, $\nu_{4}>0$, and $\nu_{5}>0$, we have, $|X \tilde{X}| \leq \frac{1}{\nu_{1}} Q(X)+$ $\nu_{1} Q(\tilde{X}),|X(t) X(t-\tau)| \leq \frac{1}{\nu_{2}} Q(X(t))+\nu_{2} Q(X(t-$ $\tau)),|X(t) \tilde{X}(t-\tau)| \leq \frac{1}{\nu_{3}} Q(X(t))+\nu_{3} Q(\tilde{X}(t-\tau))$, $|\tilde{X}(t) \tilde{U}(t-\tilde{\tau})| \leq \frac{1}{\nu_{4}} Q(\tilde{X}(t))+\nu_{4} Q(\tilde{U}(t-\tilde{\tau}))$, and $|X(t) \tilde{U}(t-\tilde{\tau})| \leq \frac{1}{\nu_{5}} Q(X(t))+\nu_{5} Q(\tilde{U}(t-\tilde{\tau})$. Consequently, $\dot{V}$ satisfies for almost all $t \geq 0$,

$$
\begin{aligned}
\dot{V}(t) \leq & -\left[2 \lambda_{1} \mathfrak{a}_{1}-\mathfrak{b}_{1}\right] Q(\tilde{X}(t))-\left[2 \mathfrak{a}_{6}-\mathfrak{b}_{2}\right] Q(X(t)) \\
& -\left[\lambda_{4} e^{-\rho_{1} \tilde{\tau}}-\mathfrak{b}_{3}\right] Q(\tilde{U}(t-\tilde{\tau}))-\rho_{1} \lambda_{4} \mathcal{Y}\left(\tilde{U}_{t}\right) \\
& -\left[\lambda_{2} e^{-\rho_{2} \tau}-\mathfrak{b}_{4}\right] Q(X(t-\tau))-\rho_{2} \lambda_{2} \mathcal{S}\left(X_{t}\right) \\
& -\left[\lambda_{3} e^{-\rho_{2} \tau}-\mathfrak{b}_{5}\right] Q(\tilde{X}(t-\tau))-\rho_{2} \lambda_{3} \mathcal{S}\left(\tilde{X}_{t}\right) \\
& -\mathfrak{a}_{5} \lambda_{4} F(X(t), \tilde{X}(t)) \tilde{U}(t-\tilde{\tau}) \\
& +X(t) G\left(X_{t}, \tilde{X}_{t}\right)+\lambda_{4} Q(F(\tilde{X}(t), X(t))) \\
& -\lambda_{4} F(X(t), \tilde{X}(t))\left[\mathfrak{a}_{4} \tilde{X}(t)+\mathfrak{a}_{2} X(t)\right],
\end{aligned}
$$

where

$$
\left\{\begin{aligned}
\mathfrak{b}_{1}= & \lambda_{3}+\lambda_{4} \mathfrak{a}_{4}^{2}+\nu_{1}\left|\mathfrak{a}_{2} \lambda_{1}+\mathfrak{a}_{7}-\lambda_{4} \mathfrak{a}_{2} \mathfrak{a}_{4}\right| \\
\mathfrak{b}_{2}= & \lambda_{2}+\lambda_{4} \mathfrak{a}_{2}^{2}+\frac{\left|\mathfrak{a}_{2} \lambda_{1}+\mathfrak{a}_{7}-\lambda_{4} \mathfrak{a}_{2} \mathfrak{a}_{4}\right|}{\nu_{1}} \\
& +\frac{\left|\mathfrak{a}_{8}\right|}{\nu_{2}}+\frac{\left|\mathfrak{a}_{9}\right|}{\nu_{3}}+\frac{\left|\mathfrak{a}_{2} \mathfrak{a}_{5}\right| \lambda_{4}}{\nu_{5}} \\
\mathfrak{b}_{3}= & \lambda_{4} \mathfrak{a}_{5}^{2}+\nu_{4}\left|\mathfrak{a}_{3} \lambda_{1}+\mathfrak{a}_{4} \mathfrak{a}_{5} \lambda_{4}\right|+\nu_{5} \lambda_{4}\left|\mathfrak{a}_{2} \mathfrak{a}_{5}\right|, \\
\mathfrak{b}_{4}= & \nu_{2}\left|\mathfrak{a}_{8}\right|, \quad \text { and } \mathfrak{b}_{5}=\nu_{3}\left|\mathfrak{a}_{9}\right|
\end{aligned}\right.
$$

For the sake of brevity, the detailed steps to follow in order to obtain sufficient decay conditions (that ensure the exponential convergence to the origin of (15)) are omitted here (see [8] for an extended version). However, to provide guidance, we specify that $\lambda_{1}, \ldots, \lambda_{4}$, $\nu_{1}, \ldots, \nu_{5}, \rho_{1}$ and $\rho_{2}$, are selected in order to guarantee that the origin of system (15) is exponentially stable, for all initial conditions belonging to the set

$$
\mathcal{B}=\left\{\left(\varphi_{X}, \varphi_{\tilde{X}}, \varphi_{\tilde{U}}\right) \in \mathcal{C}_{\tau} \times \tilde{\mathcal{C}}_{\tau} \times \tilde{\mathcal{C}}_{\tilde{\tau}} \mid V\left(\varphi_{X}, \varphi_{\tilde{X}}, \varphi_{\tilde{U}}\right)<\bar{V}\right\}
$$

where (with an abuse of notation) we consider the spaces of continuous functions, $\mathcal{C}_{\tau}=$
$\mathcal{C}\left([-\tau, 0],\left(-x_{e},+\infty\right)\right), \quad \tilde{\mathcal{C}}_{\tau}=\mathcal{C}\left([-\tau, 0],\left(-\tilde{x}_{e},+\infty\right)\right)$, $\tilde{\mathcal{C}}_{\tilde{\tau}}=\mathcal{C}\left([-\tilde{\tau}, 0],\left(-\tilde{u}_{e},+\infty\right)\right)$, and a sub-level $\bar{V}>0$ of the Lyapunov-like functional ${ }^{2}$ is defined. We emphasize that the nonlinear terms where $F$ and $G$ are involved in (24) are used only to determine a subset of the basin of attraction of the studied equilibrium. More precisely, for all initial conditions belonging to $\mathcal{B}$, we can prove that for almost all $t \geq 0$,

$$
\dot{V}(t) \leq-2 \overline{\mathfrak{d}} V\left(X_{t}, \tilde{X}_{t}, \tilde{U}_{t}\right), \text { where, } \overline{\mathfrak{d}}>0 .
$$

We integrate (26) and we obtain for all $t \geq 0$

$$
V\left(X_{t}, \tilde{X}_{t}, \tilde{U}_{t}\right) \leq e^{-2 \overline{\mathfrak{d}} t} V\left(\varphi_{X_{t}}, \varphi_{\tilde{X}_{t}}, \varphi_{\tilde{U}_{t}}\right) .
$$

Consequently, $X^{2}(t)+\lambda_{1} \tilde{X}^{2}(t) \leq 2 e^{-2 \bar{\jmath} t} V\left(\varphi_{X}, \varphi_{\tilde{X}}, \varphi_{\tilde{U}}\right)$, for all $t \geq 0$. We conclude that the trajectories $X(t)$ and $\tilde{X}(t)$ converge exponentially to the origin with a decay rate smaller or equal to $\overline{\mathfrak{d}}$. From the second equation in (15), and the inequality $2 \tilde{K}^{-\tilde{\gamma} \tilde{\tau}}<1$, we can prove that $\tilde{U}(t)$ converges exponentially to zero when $X(t)$ and $\tilde{X}(t)$ converge exponentially to the origin of the shifted system (15). The above discussion in summarized (and reformulated for the nonshifted model (2)) -for a typical selection of the weighting constants $\lambda_{i}$ and $\nu_{i^{-}}$as follows:

Theorem 1: Let us assume that the system (2) admits a strictly positive steady state $\mathfrak{D}$ (Proposition 1 ). If

$$
\begin{aligned}
& \text { i) } \quad\left(2 \tilde{K} e^{-\tilde{\gamma} \tilde{\tau}}\right)^{2}+2 \tilde{K} e^{-\tilde{\gamma} \tilde{\tau}}<1, \\
& \text { ii) } \frac{\mathfrak{b}_{1}}{4}-\tilde{\alpha} \tilde{\theta} \tilde{x}_{e}<\tilde{\beta}\left(x_{e}+\tilde{\alpha} \tilde{x}_{e}\right)+\tilde{\delta}, \\
& \text { iii) } \frac{\mathfrak{b}_{2}}{2}-\theta x_{e}<\beta\left(x_{e}+\tilde{x}_{e}\right)+\delta,
\end{aligned}
$$

where the constants $\mathfrak{b}_{i}$ and $\mathfrak{a}_{i}$ are defined in (25) and (18), then all the trajectories associated with initial conditions belonging to

$$
\begin{aligned}
& \left\{\left(\varphi_{x}, \varphi_{\tilde{x}}, \varphi_{\tilde{u}}\right) \in \mathcal{C}\left([-\tau, 0], \mathbb{R}^{+}\right) \times \mathcal{C}\left([-\tau, 0], \mathbb{R}^{+}\right)\right. \\
& \left.\quad \times \mathcal{C}\left([-\tilde{\tau}, 0], \mathbb{R}^{+}\right) \mid V\left(\varphi_{x}-x_{e}, \varphi_{\tilde{x}}-\tilde{x}_{e}, \varphi_{\tilde{u}}-\tilde{u}_{e}\right)<\bar{V}\right\}
\end{aligned}
$$

are exponentially attracted towards $\mathfrak{D}$, with a decay rate smaller than or equal to $\overline{\mathfrak{d}}$.

\section{CONCluding Discussion ANd PERsPeCtives}

Taking into account dormancy in tumor cell populations is a new trend in cancer therapy. Mechanisms underlying the dormancy of cancer stem cells, at stake in the present study, were analyzed by a coupled delay differential-difference model that describes the possible coexistence of healthy and cancer stem cells (CSCs). The first part of this model analysis allowed us to show that CSC dormancy results from complex relationships between the different biological parameters involved in the model. Theorem 1 provides sufficient local exponential stability conditions, that can be enforced in order to suggest an efficient therapeutic guideline to bring aggressive CSCs into a dormancy state, a realistic

\footnotetext{
${ }^{2}$ The functional $V$ is degenerate, since $V=0$ does not imply that $\tilde{U}=0$. This is a characteristic of differential-difference systems.
} 
therapeutic goal. Indeed, our ultimate goal is to provide biological conditions that can be interpreted as provided by a well-chosen combined targeted therapy leading to control cancer as a chronic disease. For instance, in Proposition 1, we established that $\mathfrak{D}$ exists if and only if $2 \tilde{K} e^{-\tilde{\gamma} \tilde{\tau}}<1<2 e^{-\tilde{\gamma} \tilde{\tau}}$. Satisfying this condition is obtained by increasing the product $\tilde{\gamma} \tilde{\tau}$ and decreasing $\tilde{K}$, i.e., fulfilling the inequality on the left hand side without violating the inequality on the right hand side. Increasing $\tilde{\gamma} \tilde{\tau}$ means that we extend the duration of the cell cycle $\tilde{\tau}$ (i.e., slowing it down: cytostatic drugs) and/or increase the apoptosis rate $\tilde{\gamma}$ (cytotoxic drugs). In the case of hematopoiesis, leukemic cells may be targeted by drugs such as tyrosine kinase inhibitors quizartinib (AC220) [32] or erlotinib [18] at moderate doses to increase $\tilde{\tau}$, while cytosine arabinoside can be used to increase the apoptosis rate $\tilde{\gamma}$. Similarly, we can consider acting on another target, the $\delta$ and $\tilde{\delta}$ parameters, by increasing them to enhance the differentiation rate, that is constantly blocked at some maturation stage in acute leukemias. This may be achieved by using dihydroorotate dehydrogenase inhibitors [30]. Finally, $\beta(0)$ and $\tilde{\beta}(0)$, can be increased using G-CSF molecules [13]. These are sketched principles of such a combined therapy that we will develop further in a forthcoming study ([8]).

\section{REFERENCES}

[1] M. Adimy, A. Chekroun, T. M. Touaoula. Age-Structured and Delay Differential-Difference Model Of Hematopoietic Stem Cell Dynamics, Discret And Continuous Dynamical Systems Series B, Volume 20, Number 9, pp. 2765-2791, (2015).

[2] M. Adimy, F. Crauste, A. Abdllaoui, Discrete MaturityStructured Model of Cells Differentiation with Applications to Acute Myelogenous Leukemia, J. Biological Systems, No. 3, pp. 395-424, (2008).

[3] B. Alberts, D. Bray, K. Hopkin, A. Johnson, J. Lewis, M. Raff, K. Roberts, P. Walter, Essential Cell Biology, Fourth Edition, Garland Science, pp. 865, (2013).

[4] J.L. Avila, C. Bonnet, J. Clairambault, H. Özbay, S. Niculescu, F. Merhi, A. Ballesta, R. Tang, and J.-P. Marie. Analysis of a new model of cell population dynamics in acute myeloid leukemia, In «Delay Systems : From Theory to Numerics and Applications», Advances in Delays and Dynamics, pp. 315328, Springer, (2014).

[5] H.M. Blau, T.R. Brazelton, and J.M. Weimann, The Evolving Concept Review of a Stem Cell: Entity or Function?, Cell, Vol. 105, pp. 829-841, (2001).

[6] W. Djema, F. Mazenc, C. Bonnet, Stability Analysis and Robustness Results for a Nonlinear System with Distributed Delays Describing Hematopoiesis, Systems and Control Letters, Vol. 102, pp. 93-101, (2017).

[7] W. Djema, F. Mazenc, C. Bonnet, J. Clairambault, P. Hirsch, F. Delhommeau, Stability of a Delay System Coupled to a Delay Differential-Difference System Describing the Coexistence of Ordinary and Mutated Hematopoietic Stem cells, the $55^{\text {th }}$ Conference on Decision and Control, Las Vegas, USA, (2016).

[8] W. Djema, F. Mazenc, C. Bonnet, J. Clairambault, E. Fridman, F. Delhommeau, Control and Eradication of Tumor Stem Cells: Mathematical Modeling and Stability Issues, To be submitted, (2017).

[9] H. Döhner, D.J. Weisdorf, C.D. Bloomfield, Acute Myeloid Leukemia, N. Engl. J. Med. 373, pp. 1136-1152, (2015).

[10] H. Enderling, Cancer Stem Cells and Tumor Dormancy, Sys. Biol. of Tumor Dormancy, Springer NY, pp. 55-71, (2013).

[11] T. Enver, M. Pera, C. Peterson, P.W. Andrews, Stem cell states, fates, and the rules of attraction, Cell Stem Cell 4, pp. 387-397, (2009).
[12] C. Foley, M.C. Mackey, Dynamic hematological disease: a review, J. Math. biology, 58.1-2: pp. 285-322, (2009).

[13] C. Foley, , S. Bernard, and M.C. Mackey, " Cost-effective GCSF therapy strategies for cyclical neutropenia: Mathematical modelling based hypotheses, Journal of theoretical biology 238.4: pp. 754-763, (2006).

[14] E. Fridman, C. Bonnet, F. Mazenc, W. Djema, Stability of the cell dynamics in Acute Myeloid Leukemia, Systems \& Control Letters 88, pp. 91-100, (2016).

[15] R. Hoffman, E.J. Benz, L.E. Silberstein, H. Heslop, J. Weitz, J. Anastasi, Hematology: Basic Principles and Practice, $6^{\text {th }}$ Edition, Elsevier, Churchill Livingstone, (2012).

[16] G. Jansen, R. Gatenby, C. A. Aktipis, Control vs. eradication: Applying infectious disease treatment strategies to cancer, Proceedings of the National Academy of Sciences of United States of America, vol. 112, no. 4, pp. 937-938, (2015).

[17] I. Kareva, Primary and Metastatic Tumor Dormancy as a Result of Population Heterogeneity, Biol. Dir. 37, 11:1, (2016).

[18] E. Lainey, S. Thépot, C. Bouteloup, M. Sébert, L. Adès, M. Tailler, C. Gardin, S. de Botton, A. Baruchel, P. Fenaux, G. Kroemer, S. Boehrer Tyrosine kinase inhibitors for the treatment of acute myeloid leukemia: Delineation of antileukemic mechanisms of action, Biochemical Pharmacology 82, pp. 1457-1466, (2011).

[19] J.D. Logan, Applied Partial Differential Equations, 3rd edition. Undergraduate Texts in Mathematics, Springer International Publishing Switzerland, (2015).

[20] A. G. McKendrick, Applications of mathematics to medical problems, Proceedings of the Edinburgh Mathematical Society 44, pp. 98-130, (1925).

[21] M.C. Mackey, Unified hypothesis of the origin of plastic anemia and periodic hematopoiesis, Blood 51, pp. 941-956, (1978).

[22] A. Marciniak-Czochra, T. Stiehl, A.D. Ho, W. Jäger, W. Wagner, Modeling of asymmetric cell division in hematopoietic stem cells-regulation of self-renewal is essential for efficient repopulation, Stem cells and development 18.3, pp. 377-386, (2009).

[23] D. Morgan, The Cell Cycle: Principles of Control, Primers in Biology Series, Oxford University Press, pp. 297, (2006).

[24] G.N. Naumov, J. Folkman, O. Straume, Tumor dormancy due to failure of angiogenesis: role of the microenvironment. Clin Exp Metastasis, 26(1):51-60, (2009).

[25] M. Nielsen, J. Thomsen, S. Primdahl, U. Dyreborg, J. Andersen, Breast cancer and atypia among young and middle-aged women: a study of 110 medicolegal autopsies, Br J Cancer. 56(6):814-9, (1987).

[26] H. Özbay, C. Bonnet, H. Benjelloun, J. Clairambault, Stability analysis of cell dynamics in leukemia. Math. Model Nat. Phenom., Vol. 7, No. 1, pp. 203-234, (2012).

[27] E. Passegué, C.H. Jamieson, L.E. Ailles, I.L. Weissman, Normal and leukemic hematopoiesis: Are leukemias a stem cell disorder or a reacquisition of stem cell characteristics?, Proc. Natl. Acad. Sci., Regenerative Medicine, USA 100 (Suppl 1), pp. 11842-11849, (2003).

[28] E. Pronier, F. Delhommeau, Inhibition of TET2-mediated conversion of 5-methylcytosine to 5-hydroxymethylcytosine disturbs erythroid and granulomonocytic differentiation of human hematopoietic progenitors, Blood, 118(9), pp. 2551-2555, (2011).

[29] L. Pujo-Menjouet, S. Bernard, M.C. Mackey, Long period oscillations in a $G_{0}$ model of hematopoietic stem cells, SIAM. J. Appl. Dynam. Syst. 4(2), pp. 312-332, (2005).

[30] D.B. Sykes, et al. Inhibition of dihydroorotate dehydrogenase overcomes differentiation blockade in acute myeloid leukemia, Cell, 167.1, pp. 171-186, (2016).

[31] K.P. Wilkie, P. Hahnfeldt, Tumor-immune dynamics regulated in the microenvironment inform the transient nature of immune-induced tumor dormancy, J. Cancer Research, 73(12), pp. 3534-44, (2013).

[32] P.P. Zarrinkar, AC220 is a uniquely potent and selective inhibitor of FLT3 for the treatment of acute myeloid leukemia (AML), Blood, 114(14): pp. 2984-2992, (2009). 